\title{
Competitive Facility Location along a Highway*
}

\author{
Hee-Kap Ahn ${ }^{\dagger}$ Siu-Wing Cheng ${ }^{\ddagger}$ Otfried Cheong ${ }^{\dagger}$ \\ Mordecai Golin ${ }^{\ddagger}$ René van Oostrum ${ }^{\dagger}$
}

April 10, 2001

\begin{abstract}
We consider a competitive facility location problem with two players. Players alternate placing points, one at a time, into the playing arena, until each of them has placed $n$ points. The arena is then subdivided according to the nearest-neighbor rule, and the player whose points control the larger area wins. We present a winning strategy for the second player, where the arena is a circle or a line segment. We also consider a variation where players can play more than one point at a time for the circle arena.
\end{abstract}

\section{Introduction}

The classical facility location problem [5] asks for the optimum location of a new facility (police station, super market, transmitter, etc.) with respect to a given set of customers. Typically, the function to be optimized is the maximum distance from customers to the facility - this results in the minimum enclosing disk problem studied by Megiddo [8], Welzl [12] and Aronov et al. [2].

Competitive facility location deals with the placement of sites by competing market players. Geometric arguments are combined with arguments from game theory to see how the behavior of these decision makers affect each other. Competitive location models have been studied in many different fields, such as spatial economics and industrial organization [1,9], mathematics [6] and operations research $[3,7,11]$. Comprehensive overviews of competitive facility locations models are the surveys by Friesz et al. [11], Eiselt and Laporte [3] and Eiselt et al. [4].

We consider a model where the behavior of the customers is deterministic in the sense that a facility can determine the set of customers more attracted to it than to any other facility. This set is called the market area of the facility. The collection of market areas forms a tessellation of the underlying space. If customers choose the facility on the basis of distance in some metric, the tessellation is the Voronoi Diagram of the set of facilities [10].

We address a competitive facility location problem that we call the Voronoi Game. It is played by two players, Blue and Red, who place a specified number, $n$, of facilities in a region $U$. They alternate placing their facilities one at a time, with Blue going first. After all $2 n$ facilities have been placed, their decisions are evaluated by considering the Voronoi diagram of the $2 n$ points. The player whose facilities control the larger area wins.

\footnotetext{
${ }^{*}$ Part of the work was done while the first, third and fifth authors are at the Department of Computer Science, HKUST, Hong Kong.

${ }^{\dagger}$ Department of Computer Science, Utrecht University, Netherlands. Email: \{heekap, otfried, rene\}@cs.uu.nl

${ }_{\ddagger}^{\ddagger}$ Department of Computer Science, HKUST, Hong Kong. Email: \{scheng, golin\}@cs.ust.hk
} 
More formally, let $\left\{b_{i}\right\}_{i=1}^{n}$ and $\left\{r_{i}\right\}_{i=1}^{n}$ be the respective locations of the blue and red points and set

$$
\begin{aligned}
\mathcal{B} & =\left|\left\{u \in U: \min _{i} d\left(u, b_{i}\right)<\min _{i} d\left(u, r_{i}\right)\right\}\right|, \\
\mathcal{R} & =\left|\left\{u \in U: \min _{i} d\left(u, r_{i}\right)<\min _{i} d\left(u, b_{i}\right)\right\}\right|
\end{aligned}
$$

where $d(u, v)$ is an underlying metric and $|\cdot|$ indicates the area of a set. Blue wins if and only if $\mathcal{B}>\mathcal{R}$, Red wins if and only if $\mathcal{R}>\mathcal{B}$ and the game ends in a tie if $\mathcal{B}=\mathcal{R}$.

The most natural Voronoi Game is played in a two-dimensional arena $U$ using the Euclidean metric. Unfortunately nobody knows how to win this game, even for very restricted regions $U$. In this note we present strategies for winning one-dimensional versions of the game, where the arena is a circle or a line segment, and variations. In other words, we consider competitive facility location along an Australian highway.

The next section discusses the simplest game, on the circle. It is obvious that the second player, Red, can always achieve a tie by playing on the antipode of Blue's move. One might try to tweak this strategy such that it results in a win for Red. This doesn't seem to work, and we present instead a quite different winning strategy for Red.

Section 3 describes how this strategy remains a winning strategy even if the rules of the game are drastically relaxed.

In Section 4 we finally turn to the line segment arena. It would appear that Blue has an advantage here, because it can play the midpoint of the segment in its first move. We show that this doesn't help, and prove that Red still has a winning strategy. The strategy is quite similar to the one for the circle case, but its analysis (because of a loss of symmetry) is more detailed.

\section{The basic circle game}

There are two players, Blue and Red, each having $n$ points to play, where $n>1$. They alternate placing these points on circle $C$, with Blue placing the first point, Red the second, Blue the third, etc., until all $2 n$ points are played. We assume that points cannot lie upon each other. Let $\left\{b_{i}\right\}_{i=1}^{n}$ be the locations of the blue points and $\left\{r_{i}\right\}_{i=1}^{n}$ be those of the red ones. After all of the $2 n$ points have been played each player receives a score equal to the total circumference of the circle that is closer to that player than to the other, i.e., Blue and Red have respective scores

$$
\begin{aligned}
\mathcal{B} & =\left|\left\{x \in C: \min _{i} d\left(x, b_{i}\right)<\min _{i} d\left(x, r_{i}\right)\right\}\right| \\
\mathcal{R} & =\left|\left\{x \in C: \min _{i} d\left(x, r_{i}\right)<\min _{i} d\left(x, b_{i}\right)\right\}\right|
\end{aligned}
$$

The player with the highest score (the larger circumference) wins.

The question that we address here is, Does either player have a winning strategy and, if yes, what is it? We will see below that the second player, Red, always has a winning strategy.

Before giving the strategy we introduce some definitions. We parameterize the circle using the interval $[0,1]$, where the points 0 and 1 are identified. Arcs on the circle are written as $[x, y]$ implying the clockwise arc running from $x$ to $y$, as in $[.5, .6]$ or $[.9, .1]$.

Definition 1 The $n$ points $u_{i}=\frac{i}{n}, i=0,1, \ldots, n-1$ are keypoints. 


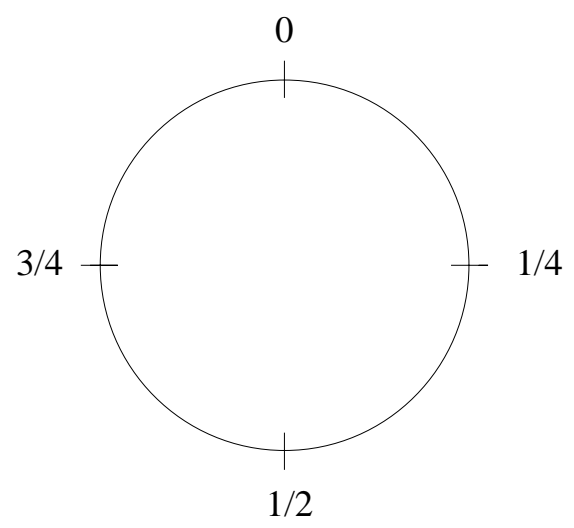

Figure 1: There are four keypoints when $n=4$.

Figure 1 shows the keypoints for $n=4$.

We call an arc between two clockwise consecutive red/blue points an interval. The interior of an interval is free of red/blue points. At any given time during the game the circle is partitioned into intervals. An interval is monochromatic if its endpoints have the same color, and bichromatic if they have different colors. A blue interval is a blue monochromatic one, a red interval a red monochromatic one. We denote the total length of all red intervals by $R_{m}$, and the total length of all the blue intervals by $B_{m}$. An interval is called a key interval if both of its endpoints are keypoints.

The important thing to notice is that at the end of the game the length of each bichromatic interval is divided equally among the two players, so $\mathcal{R}-\mathcal{B}=R_{m}-B_{m}$ and Red wins if and only if $R_{m}>B_{m}$. We devise our strategy to force this to happen.

Since we can parameterize the circle arbitrarily, we can assume without loss of generality that Blue plays his first point on 0 and thus on a keypoint. We now describe Red's winning strategy. Figure 2 shows an example.

\section{Red's Keypoint Strategy}

Stage I: If there is an empty keypoint then Red plays onto the keypoint.

Stage I ends after the last keypoint is played (by either Red or Blue).

Stage II: If there is no empty keypoint and it is not Red's last move then Red plays her point into a largest blue interval. We call this breaking the blue interval.

Stage II ends when Blue plays his last point.

Stage III: Red's last move. There are two possibilities:

(i) if there exists more than one blue interval then Red breaks a largest one by placing her point inside.

(ii) if there is only one blue interval define $\ell<\frac{1}{n}$ to be its length. Red's move is to go to a bichromatic key interval and claim a red interval of length larger than $\ell$ by placing a red point closer than $\frac{1}{n}-\ell$ to the blue endpoint of the bichromatic key interval.

The two following lemmas will be needed. 


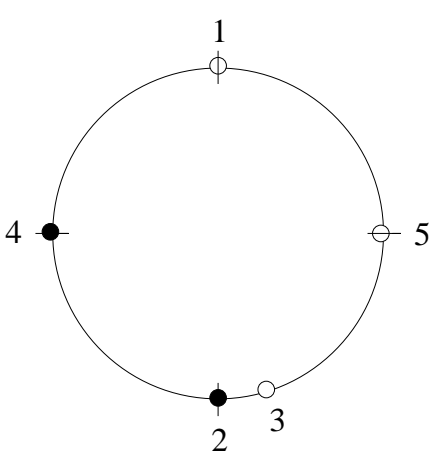

Stage I

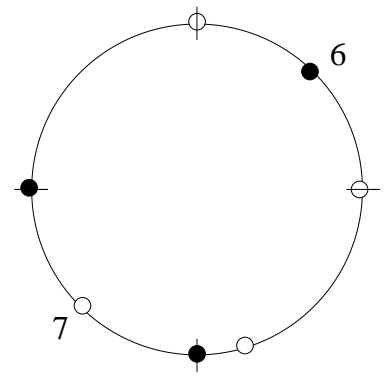

Stage II

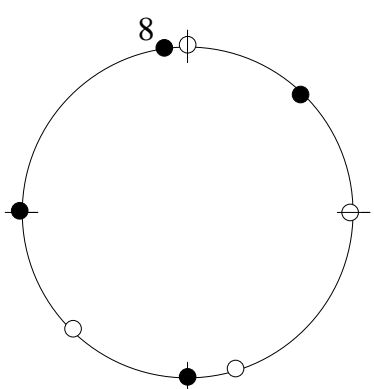

Stage III (ii)

Figure 2: There are four points to be played for both Blue and Red. The white dots represent Blue's points and the black dots represent Red's points. We label the dots in chronological order.

Lemma 1 Let $B$ be a set of b blue points and let $R$ be a set of $r$ red points currently on the circle with $b \geq r$. Let $n(R)$ be the number of red intervals they form and $n(B)$ the number of blue ones. Then $n(B)-n(R)=b-r$.

Proof: The proof will be by induction on $r$. If $r=0$ then $b$ blue points form $b$ blue intervals so $n(B)=b, n(R)=0$ and the condition $n(B)-n(R)=b-r$ is satisfied.

Now suppose that the lemma is true for all configurations of $b$ blue points and $r-1$ red ones. Deleting any red point $p$ from $R$ leaves $b$ blue and $r-1$ red points, so $n(B)-n(R-\{p\})=b-r+1$. We now add the red point $p$ back into the configuration and ask how the monochromatic intervals can change. There are three possible placements of $p$ :

(i) inside a red interval, increasing $n(R)$ by one and leaving $n(B)$ unchanged.

(ii) inside a blue interval, decreasing $n(B)$ by one and leaving $n(R)$ unchanged.

(iii) inside a bichromatic interval, increasing $n(R)$ by one and leaving $n(B)$ unchanged.

After all three of these cases we find that $n(B)-n(R)=n(B)-n(R-\{p\})-1=b-r$.

Lemma 2 Suppose that all $n$ keypoints are covered and Blue has just moved (possibly covering the last keypoint). If there is only one blue interval and this interval has length $<\frac{1}{n}$, then there exists a bichromatic key interval.

Proof: We apply the pigeon hole principle: At most $2 n-1$ points have been played, $n$ of them on keypoints. Consider the $n$ circle arcs of length $\frac{1}{n}$ formed by the $n$ keypoints. Since the blue interval has length $<\frac{1}{n}$, at least one of its endpoints is inside an arc. That leaves only $n-2$ points to have been played inside the $n-1$ remaining arcs. Therefore, one of the arcs must be free of points, forming a key interval. Since there is only one blue interval, there is no red interval by Lemma 1 . Therefore, this key interval is bichromatic.

Theorem 1 The keypoint strategy is a well-defined winning strategy for Red.

Proof: We start with a simple observation. Since the circle contains only $n$ keypoints and Blue's first move covers the first keypoint, Red will play onto at most $n-1$ keypoints. Thus Stage I always ends before Red plays her last point. 
Consider Stage II. Lemma 1 implies that after each play by Blue $(b=r+1)$ there is always at least one blue interval on the circle, so Stage II of the strategy is indeed well defined.

We make two observations concerning the situation after Stage II, when Red has played her $n-1$ 'st point. The first is that there is no blue key interval. Let $k$ be the number of keypoints played by Blue during the game. Red has covered the remaining $n-k$ keypoints by the end of Stage I. If $k=1$ (the only case in which Red skips Stage II), then there certainly is no blue key interval as there is only one blue keypoint. When $k>1$, Blue can define at most $k-1$ blue key intervals with its $k$ keypoints (since Red has at least one keypoint). Note that since all keypoints are played by the end of Stage I, all intervals in Stages II and III have length at most $\frac{1}{n}$. In particular a blue key interval is longer than any other blue interval. Since Red plays $k-1$ points in Stage II, all blue key intervals are broken during Stage II.

The second observation concerning the situation after stage II is that all red intervals are key intervals. This statement is true at the end of Stage I, as Red has so far only played onto keypoints, and all keypoints are covered. During Stage II, Red uses her points to break blue intervals, and therefore creates bichromatic intervals only. Blue cannot create red intervals, and so, at the end of Stage II, all red intervals are indeed key intervals.

We now show why Stage III is well defined and why Red wins. Suppose that Blue has just played his last point and it is now time for Stage III, Red's last move. From Lemma 1 we know that $n(B) \geq 1$.

If $n(B)>1$ before Red's last move then the strategy is well defined: Red breaks a largest blue interval. This decreases $n(B)$ by 1 so the game ends with $n(B) \geq 1$. By Lemma 1 we have $n(R)=n(B) \geq 1$. But now note that from the observations in the preceding paragraphs all existing red intervals are key intervals while all existing blue intervals have length strictly less than $\frac{1}{n}$. Since all red intervals are longer than all blue intervals and there are the same number of red ones and blue ones we find that $R_{m}>B_{m}$ and Red wins.

If $n(B)=1$ before Red's last move the strategy requires that the unique blue interval has length $\ell<\frac{1}{n}$, and that there exists a bichromatic key interval. The first fact was already observed above, the second fact follows from Lemma 2 .

After Red places her last point Blue still has one blue interval of length $\ell$ while Red has one red interval of length $>\ell$. Thus $R_{m}>\ell=B_{m}$ and Red wins.

\section{A modified circle game}

The basic game can be modified in many different ways. The simplest modification allows the players to play more than one point at a time. More complicated modifications permit the players (both or one) to choose before each turn, how many points they play.

Suppose that there are $k \leq n$ rounds. Let $\beta_{i}$ and $\gamma_{i}$ be the numbers of points that Blue and Red play respectively in round $i$. Suppose that the following restrictions are placed.

- $\forall 1 \leq i \leq k, \beta_{i}, \gamma_{i}>0$.

- $\forall 1 \leq j \leq k, \sum_{i=1}^{j} \beta_{i} \geq \sum_{i=1}^{j} \gamma_{i}$.

- $\sum_{i=1}^{k} \beta_{i}=\sum_{i=1}^{k} \gamma_{i}=n$.

- $\beta_{1}<n$. 
Then Red still wins by following exactly the same strategy as in the previous section of first filling in the keypoints and then breaking the largest blue intervals until Red plays it's last point when it follows the Stage III rules. The proof that the strategy is well defined and wins is almost exactly the same as the one in the previous section so we will not repeat it here.

Note that this generalization includes both the original game and the "batched" version in which each player plays the same number $(>1)$ of points at each turn. Note, too, that $k, \beta_{i}$, and $\gamma_{i}$ need not be fixed in advance. For example, Blue may decide at every move how many points he will play and then Red plays the same number.

We conclude this section by noting that the condition $\beta_{1}<n$ is essential since otherwise Blue would play the keypoints, forcing a tie.

\section{The line segment version}

We now move on to the version of the game played on a line segment. We consider it to be horizontal and parameterized as $[0,1]$. The scoring is the same as in the basic circle game except that the player with the leftmost point claims everything between 0 and the point, and the player with the rightmost point claims everything between the point and 1 . We assume that $n>1$, and points cannot lie upon each other. When $n=1$, Blue wins by placing onto $\frac{1}{2}$.

We modify some of the old definitions and introduce new ones:

Definition 2 The $n$ points $u_{i}=\frac{1}{2 n}+\frac{i}{n}, i=0,1, \ldots, n-1$ are keypoints.

The left segment is the segment from 0 to the leftmost red or blue point. The right segment is the segment from the rightmost red or blue point to 1 . The border interval is the union of the left and right segments. An interval is a section of the line segment with red/blue endpoints and no red/blue points in its interior. We consider the border interval an interval. An interval, including the border interval, is monochromatic if its endpoints have the same color, and bichromatic if they have different colors. With this definition of intervals, Lemmas 1 and 2 are true for the line segment as well.

We denote the total length of all of the blue intervals - including, if appropriate, the border interval - by $B_{m}$, the total length of all of the red intervals - again including, if appropriate, the border interval- by $R_{m}$. When the border interval is bichromatic, we use $B_{b}$ to denote the length of the left/right segment with a blue endpoint and $R_{b}$ to denote the length of the left/right segment with a red endpoint. If the border interval is monochromatic, then $B_{b}=R_{b}=0$. Since all bichromatic non-border intervals are equally shared by both players $\mathcal{R}-\mathcal{B}=\left(R_{m}+R_{b}\right)-\left(B_{m}+B_{b}\right)$ and, as in Section 2, we design our strategy so that Red finishes with the right hand side of the equation $>0$.

We now introduce the line strategy, a modified version of the circle strategy. Figure 3 shows an example.

\section{Red's Line strategy}

Stage I: If there is an empty keypoint then Red plays the keypoint. If $u_{0}$ or $u_{n-1}$ have not yet been played then Red should play onto one of them first. Stage I ends after the last keypoint is played by either Red or Blue. Note that the game may finish in Stage I. 


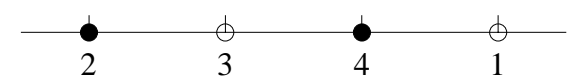

Stage I

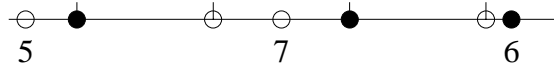

Stage II

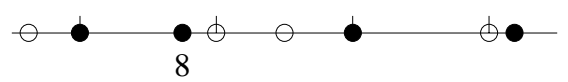

Stage III

Figure 3: There are four points to be played for both Blue and Red. The white dots represent Blue's points and the black dots represent Red's points. We label the dots in chronological order.

Stage II: If there is no empty keypoint and it is not Red's last move then

(i) if there exists at least one blue non-border interval, then Red should break a largest blue non-border interval by placing her point inside.

(ii) if the border interval is the only blue interval, then there are two possible cases:

(a) One of the blue endpoints of the blue border interval is a keypoint:

Without loss of generality assume that it is $u_{0}$ (the other case is symmetric) and the other endpoint is $1-\ell$. From Stage I the other endpoint cannot be the keypoint $u_{n-1}$ so $\ell<\frac{1}{2 n}$. Red now places her new point at $x$ where $x$ is anywhere in $\left(\ell, u_{0}\right)$.

(b) Neither of the endpoints of the border interval are keypoints: Let $\ell$ be the length of the blue border interval; $\ell<\frac{1}{n}$. There must then exist a bichromatic key interval (Lemma 2). Red places her new point in that interval to form a new red interval of length $>\ell$.

Stage II ends after Blue plays his last point.

Stage III: If Red is placing her last point, we have two mutually exclusive cases:

(i) if there exists more than one blue interval, then Red should break a largest non-border one.

(ii) if there exists only one blue interval, then let its length be $\ell$; we will see below that $\ell<\frac{1}{n}$. Red should go to a bichromatic key interval (one will exist from Lemma 2) and claim a red interval of length $>\ell$ as follows.

- If the bichromatic key interval is not the border one, Red can do this by creating a new red interval of length $>\ell$.

- If the bichromatic key interval is the border one, then Red already possesses $\frac{1}{2 n}$ of it because it has all of either $\left[0, u_{0}\right]$ or $\left[u_{n-1}, 1\right]$. Red can therefore go to the other side it does not possess, and grab enough length to have a red border interval of length $>\ell$.

Theorem 2 The line strategy is a well-defined winning strategy for Red. 
Proof: Note that this strategy differs in at least one major aspect from the circle strategy: since we have lost circular symmetry it cannot be guaranteed that Blue plays onto at least one keypoint, and so it is possible that the game will end in Stage I, with Red playing all $n$ keypoints. In this case, all red intervals (including, possibly, the border interval) are key intervals and all blue intervals have length $<\frac{1}{n}$. By Lemma 1 Blue and Red have the same number of monochromatic intervals, so $B_{m}<R_{m}$. If the border interval is monochromatic, then $B_{b}=R_{b}=0$ and Red wins. If the border interval is bichromatic, then one of its endpoints must be the red point $u_{0}$ or $u_{n-1}$. This implies that $B_{b}<R_{b}=\frac{1}{2 n}$, and Red wins.

In what follows we may therefore assume that Blue plays onto at least one keypoint during the game. We will show that at the end of the game it will always be true that $B_{m}<R_{m}$ and, if the border interval is bichromatic, then $B_{b} \leq R_{b}$. The theorem will follow.

First note that under this assumption Stage I always ends with all keypoints covered, and Stage III is reached. Note further that Red's first move is onto either $u_{0}$ or $u_{n-1}$.

We consider Stage II. After Blue's every move there exists at least one blue interval (possibly the border interval) by Lemma 1. If there is only one such blue interval, there is no red interval. Thus, one of the two conditions (i) or (ii) of Stage II holds. The strategy is clearly well defined in cases (i) and (ii)(a), the validity of (ii)(b) follows from Lemma 2.

We will need one more observation.

Lemma 3 After Blue's last move, there is no blue key interval.

Proof: Let $k$ be the number of keypoints played by Blue, where $1 \leq k \leq\left\lceil\frac{n}{2}\right\rceil$. There are therefore at most $k-1$ blue key intervals after Stage I. Red occupies $n-k$ keypoints in Stage I, and so Stage II lasts for $k-1$ rounds. This is sufficient for all blue key intervals to be broken, since a blue key interval is longer than any other blue interval in Stage II (i) and Stage II (ii)(a).

We now prove that Red wins. There are two cases.

Lemma 4 Assume that case (ii) of Stage II never occurs. Then all red intervals are key intervals after Blue's last move.

Proof: After Stage I all red intervals are key intervals, since Red has only played keypoints and all keypoints are covered. During Stage II Red uses all her points to break blue intervals (since case (ii)(b) does not occur), and so creates only bichromatic intervals. As Blue cannot create red intervals, all red intervals remaining after Stage II are indeed key intervals.

We examine the result of Stage III, assuming that case (ii) of Stage II did not occur. Assume first that Red plays case (i) of Stage III. There are then equal numbers of red and blue intervals left after the last move. Since all blue intervals have length $<\frac{1}{n}$ by Lemma 3 , and all red intervals are key intervals by Lemma 4 , we have $B_{m}<R_{m}$. If the border interval is monochromatic, then $B_{b}=R_{b}=0$ and Red wins. If the border interval is bichromatic, then its red endpoint must be $u_{0}$ or $u_{n-1}$ (since Red plays case (i) of Stage II only), and so $B_{b} \leq R_{b}=\frac{1}{2 n}$ and Red wins.

On the other hand, assume now that Red plays case (ii) of Stage III. Then Blue has total length $\ell$, Red has $>\ell$, and so Red wins.

We now consider the remaining case, where case (ii) of Stage II does occur. 
Lemma 5 Assume that case (ii) of Stage II occurs at least once. After Red's last move in Stage II, there is no blue non-border interval and $\mathcal{B}<\mathcal{R}$.

Proof: We prove that the statement is true after the last occurrence of case (ii) in Stage II, and after each subsequent move by Red.

Consider the last occurrence of case (ii). Before Red's move, the border interval is the only blue interval.

If Red plays case (ii)(a), there is no blue interval at all after Red's move. We have then $B_{m}=R_{m}=0$ and $B_{b}<R_{b}$, which implies $\mathcal{B}<\mathcal{R}$.

If Red plays case (ii)(b), Red claims a red interval longer than the blue border interval. So after Red's move we have $\mathcal{B}<\mathcal{R}$, as $B_{m}<R_{m}$ and $B_{b}=R_{b}=0$.

Consider now the remaining moves of Stage II. In all these moves Red plays case (i), and so we can deduce that Blue uses his move to create a new blue non-border interval. Red immediately breaks this blue interval. This leaves $B_{m}, R_{m}, B_{b}$, and $R_{b}$ unchanged and destroys the only blue non-border interval. The claim therefore remains true after each subsequent move by Red, and in particular after Red's last move in Stage II.

We consider the situation right before Blue's last move. By Lemma 5, there is no blue nonborder interval, and $\mathcal{B}<\mathcal{R}$.

If the border interval is blue before Blue's last move and Blue uses that move to create a blue non-border interval move, Red plays case (i) of Stage III. This breaks the new blue non-border interval, returning $\mathcal{B}$ and $\mathcal{R}$ to their state before Blue's last move, and so Red wins.

In all other cases there is a single blue interval after Blue's last move. It cannot be a key interval by Lemma 3, and so, by Lemma 2, there is a bichromatic key interval. Red claims a red interval longer than the blue interval, and wins.

\section{Conclusions}

We have given strategies for one-dimensional competitive facility location, allowing the second player, Red, to win. The margin by which Red wins is very small, however, and in fact Blue can make it as small as he wants. Is there a strategy that would allow Red to win by a larger margin? The answer is no- our arguments can easily be transformed into a strategy that allows the first player, Blue, to lose by an arbitrarily small margin.

Do our findings have any bearing on the two-dimensional Voronoi Game? The concept of keypoints turned out to be essential to our strategies. We have seen that a player governing all keypoints cannot possibly lose the game. Is there a similar concept in two dimensions? A natural attempt would be to define a uniform square grid of keypoints. Perhaps surprisingly, a player governing this grid can still lose the game by a considerable margin.

\section{Acknowledgments}

The authors would like to thank Dr. Jacob Ecco for initially introducing them to this problem. We also thank Sunil Arya, Mark de Berg, and Joachim Gudmundsson for helpful discussions. 


\section{References}

[1] S.P. Anderson. Equilibrium existence in a linear model of spatial competition. Economica, 55:479-491, 1988.

[2] B. Aronov, M. van Kreveld, R. van Oostrum, and K. Varadarajan. Facility location on terrains. In K.Y. Chwa and Oscar H. Ibarra, editors, Proc. 9th International Symposium of Algorithms and Computation, volume 1533 of Lecture Notes Comput. Sci., pages 19-28. Springer-Verlag, 1998.

[3] H.A. Eiselt and G. Laporte. Competitive spatial models. European Journal of Operational Research, 39:231-242, 1989.

[4] H.A. Eiselt, G. Laporte, and J.-F. Thisse. Competitive location models: A framework and bibliography. Transportation Science, 27:44-54, 1993.

[5] R.L. Francis and J.A. White. Facility Layout and Location. Prentice-Hall, Inc., 1974.

[6] S.L. Hakimi. Location with spatial interactions: Competitive location and games. In R.L. Francis and P.B. Mirchandani, editors, Discrete Location Theory, pages 439-478. Wiley, New York, 1990.

[7] M. Labbé and S.L. Hakimi. Market and locational equilibrium for two competitors. Operations Research, 39:749-756, 1991.

[8] N. Megiddo. Linear-time algorithms for linear programming in $R^{3}$ and related problems. SIAM J. Comput., 12:759-776, 1983.

[9] A. Okabe and M. Aoyagy. Existence of equilibrium configurations of competitive firms on an infinite two-dimensional space. Journal of Urban Economics, 29:349-370, 1991.

[10] F. P. Preparata and M. I. Shamos. Computational Geometry: An Introduction. Springer-Verlag, New York, NY, 1985.

[11] R.L. Tobin T.L. Friesz and T. Miller. Existence theory for spatially competitive network facility location models. Annals of Operations Research, 18:267-276, 1989.

[12] Emo Welzl. Smallest enclosing disks (balls and ellipsoids). In H. Maurer, editor, New Results and New Trends in Computer Science, volume 555 of Lecture Notes Comput. Sci., pages 359-370. Springer-Verlag, 1991. 